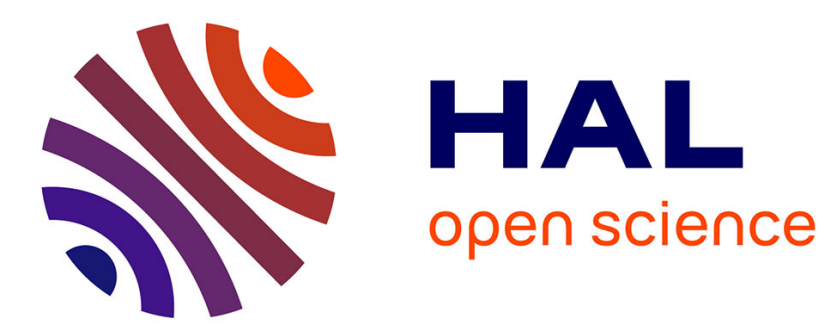

\title{
Optical diffraction of stretched lacunary spring networks
}

Pascal Monceau, Jean-Claude Lévy, Hervé Isambert, Denise Berger

\section{To cite this version:}

Pascal Monceau, Jean-Claude Lévy, Hervé Isambert, Denise Berger. Optical diffraction of stretched lacunary spring networks. Journal de Physique I, 1993, 3 (9), pp.1891-1899. 10.1051/jp1:1993219 . jpa-00246837

\section{HAL Id: jpa-00246837 https://hal.science/jpa-00246837}

Submitted on 1 Jan 1993

HAL is a multi-disciplinary open access archive for the deposit and dissemination of scientific research documents, whether they are published or not. The documents may come from teaching and research institutions in France or abroad, or from public or private research centers.
L'archive ouverte pluridisciplinaire HAL, est destinée au dépôt et à la diffusion de documents scientifiques de niveau recherche, publiés ou non, émanant des établissements d'enseignement et de recherche français ou étrangers, des laboratoires publics ou privés. 


\title{
Optical diffraction of stretched lacunary spring networks
}

\author{
Pascal Monceau $\left({ }^{1}\right)$, Jean-Claude Serge Lévy $\left({ }^{1}\right)$, Hervé Isambert $\left({ }^{2}\right)$ and Denise \\ Berger $\left({ }^{2}\right)$ \\ (') Laboratoire de Magnétisme des Surfaces, Université Paris 7, 75251 Paris 05, France \\ (2) Laboratoire d'Optique Physique, ESPCI, 10 rue Vauquelin 75005 Paris, France \\ (Received 20 January 1993, revised 6 May 1993, accepted 14 May 1993)
}

\begin{abstract}
Résumé. - Un réseau aléatoire de sites reliés par des ressorts est étiré; on en étudie analytiquement et expérimentalement le schéma de diffraction optique. Certaines régions sont simplement déplacées en bloc et leurs déplacements relatifs sont mesurés par diffraction, d'autres régions sont déformées uniformément et cette déformation est détectée optiquement. La reconstruction d'image dans l'espace réel avec filtrage dans l'espace réciproque permet de localiser des défauts typiques. On considère aussi des milieux fractals aléatoires.
\end{abstract}

\begin{abstract}
A network of randomly occupied sites connected by springs is submitted to an external stretch ; the optical diffraction pattern of the distorted network is experimentally and analytically studied. Some regions are just shifted as a whole and related shifts are measured by means of diffraction, some regions are uniformly distorted and this distortion is optically detected. The techniques of pattern reconstruction in real space by filtering in Fourier space enable typical defects to be localized. Fractal random media are also considered.
\end{abstract}

\section{Introduction.}

Elasticity in lacunary materials, or binary materials which are fractals, or randomly distributed materials, has a large interest because of numerous practical applications such as rocks and cements [1] or textile fibers [2] for instance. Yet, due to the lack of translational invariance, there is a real theoretical difficulty involved in such elastic properties. An example of this difficulty is given by the existence of ultraslow relaxation processes which have been observed both experimentally [3] and numerically [4], while a complete analytic resolution remains impossible. The preliminary example of relaxation already points out that a careful experimental investigation is required when dealing with elasticity in lacunary materials. Optical diffraction and more generally diffraction experiments with $\mathrm{X}$-rays, electrons or neutrons have the advantage of being a study of the medium as a whole which gives an interesting global response in the case of a uniform elastic stimulus as stretch or tension.

In the case of a lacunary spring network which is the simplest physical site network, also called central force model, there is no transverse elasticity at first order, i.e. atoms which are not connected by a full line of occupied nearest neighbour sites are elastically independent 
$[4,5]$. Thus in such a bidimensional stressed lacunary network, four kinds of elastic region appear :

- region I (first kind) is elastically connected to only one moving boundary, the left one ;

- region II (second kind) is only connected to the other moving boundary, the right one ;

- region III (third kind) is not connected to any moving boundary ;

- and region IV (fourth kind) is fully connected to both moving boundaries. In this paper, slipping distances between undistorted regions and affinity parameter in the distorted region are deduced from the observation of the diffraction pattern of the whole sample. Moreover, since the bidimensional distorted networks are derived from a precise numerical relaxation process [4], non-linear effects are also observed on diffraction patterns and can be linked to the concept of elastic defects in inhomogeneous materials. These measurements and evidence for local defects are the goals of this paper which deals with optical diffraction. The results can be easily translated for X-ray or electron diffraction.

A brief first section is devoted to the introduction of the elasticity problem in lacunary spring networks, while the second section deals with diffraction patterns observed from these targets under stress.

\section{Elasticity in lacunary spring networks.}

Here, two kinds of site networks at rest are considered :

- random networks on a square lattice : $R(n, q)$ where $p=n^{2}-q\left(p \leqslant n^{2}\right)$ occupied sites are randomly chosen among the $n^{2}$ ones of a full square of side length $n$,

- random Sierpinski carpets $C(n, p)$ [6-8] where each selected square is segmented into $n \times n$ subsquares, conserving out of them only $p\left(\leqslant n^{2}\right)$ randomly selected subsquares at the next step of iteration; at this step, subsquares are now considered as squares and so on. At each step of iteration the selections of $p$ subsquares are independent. Stopping the iterative process at a step $k$ of iteration, we introduce the obvious notation $C(n, p ; k)$ for such unachieved random Sierpinski carpets. Unachieved random Sierpinski carpets $C(n, p ; k)$ present both a high level of scale invariance and a large number of distinct realizations, i.e. a high level of entropy [8] which is their interesting property. Some examples of $R(n, q)$ and $C(n, p ; k)$ are given in figure 1.

a)

frac1613 avant relaxation b)

rand 250 ovant reloxation

Fig. 1. - Two sets of diffracting pupils : a) an unachieved random Sierpinski network $C(4,13 ; 2)$ and b) a random network $R(16,6)$. 
Then, in each case, for random networks $R(n, q)$ or for random Sierpinski carpets $C(n, p ; k)$, a spring of stiffness $k$ is introduced between each pair of neighbouring sites $i$ and $j$, i.e. distant of $a$ at rest, with the local Hamiltonian $H_{i j}$.

$$
H_{i j}=\frac{1}{2} k\left(\left\|\mathbf{r}_{\imath}-\mathbf{r}_{\jmath}\right\|-a\right)^{2}
$$

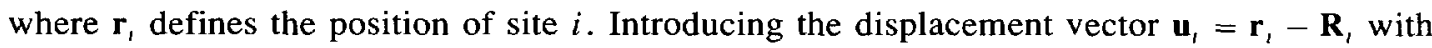
respect to the rest position $\mathbf{R}_{1}$ and the parallel displacement $\left(\mathbf{u}_{1}-\mathbf{u}_{3}\right)_{\|}$which is the projection of the displacement vector $\left(\mathbf{u}_{i}-\mathbf{u}_{j}\right)$ onto the vector $\mathbf{R}_{l}-\mathbf{R}_{J}$ at rest and of length $a$, the first nonconstant terms in the series development of the total Hamiltonian $H$ are quadratic in parallel displacements only:

$$
\begin{gathered}
H=\frac{1}{2} k \sum\left(\mathbf{u}_{\imath}-\mathbf{u}_{\jmath}\right) \|^{2}+\frac{1}{2} k \sum \frac{\left(\mathbf{u}_{i}-\mathbf{u}_{j}\right)_{\perp}^{2}\left\|\left(\mathbf{u}_{l}-\mathbf{u}_{j}\right)_{\|}\right\|}{a}\left(1-\frac{\left\|\left(\mathbf{u}_{1}-\mathbf{u}_{j}\right)_{\|}\right\|}{a}\right)+ \\
+\frac{1}{2} k \sum \frac{\left(\mathbf{u}_{i}-\mathbf{u}_{\jmath}\right)_{\perp}^{4}}{a^{2}} \\
H=H{ }^{(1)}+H_{\perp}^{(2)}+H_{\perp}^{(3)}
\end{gathered}
$$

As a consequence, the harmonic elastic coupling is restricted to sites connected by a full line of nearest neighbours, which defines a connection with independent blocks of sites ; in the case of a unidirectional displacement according to a dense direction of atoms, these independent blocks are only full segments parallel to the displacement. The stretch is imposed and defined by a given displacement of sites which are located on two opposite edges of the basic square for $R(n, q)$ or $C(n, p ; k)$. Then the state of equilibrium of the network is reached after a relaxation process which is simulated by means of a numerical overdamped relaxation process $[9,10]$. This process consists in calculating the forces $F_{1}$ on each site and displacing each bulk atom by a displacement $\mathbf{u}_{i}=\lambda \mathbf{F}$, where $\lambda$ is a convenient damping parameter [4]. The total elastic energy $E$ of the system is calculated after each run over all free sites, and such runs are repeated until the energy $E$ is left practically constant. There is an optimal choice of $\lambda$ in order to obtain a rapid convergence without chaos. Some typical spring networks are shown in figure 2 after a damped relaxation under stress. The behaviour of independent

a) fract613 apres relaxation b)

rand250 cpres reloxation 0.30

Fig. 2. - The two sets of figure 1 after relaxation under stress : a) $C(4,13 ; 2)$ with a shift of $0.3 a$ on two external sides and b) $R(16,6)$ with the same shift of $0.3 a$ on two external sides. 
segments can be noticed in this figure. This independence clearly appears on the typical displacement vector field $\mathbf{u}_{(1)}$ given in figure 3 , where horizontal lines 3,9 and 15 are regions of the fourth kind, while segments of the three other kinds appear in the horizontal lines 4,10 , 13 and 14. As expected from the previous comments about the Hamiltonian, these distorted networks exhibit all four kinds of regions which were defined in the introduction. It must be noticed that the weak transverse couplings induce weak corrections to this definition of four kinds of blocks, for instance the central segment of the horizontal line 13 in figure 3 is of the third kind and exhibits visible transverse displacements. These weak displacements are correlated over short distances only because of the weak transverse coupling and thus contribute to local defects, i.e. quite localized singularities. They also induce second order, i.e. negligible, longitudinal displacements. However the quite interesting displacement vector field is not directly observable.

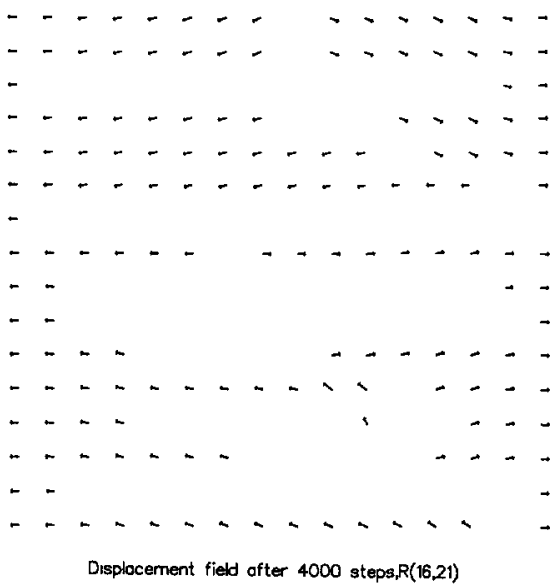

Fig. 3. - The displacement field vector after 4000 steps of iteration in a random network $R(16,21)$ with a shift of $0.3 a$ on two external sides.

\section{Diffraction on stretched lacunary spring networks.}

In the classical Fraunhofer method [11] optical diffraction by photographs of free or stretched lacunary spring networks is realized by taking as a target a copy of a bidimensional network illuminated under normal incidence by an extended laser beam. The diffracted pattern is observed in the focal plane FT (Oxy) of a lens of focal distance $f$ as shown in figure 4, practically the image in plane FT is projected onto screen $S$ by means of a lens which enables an enlargement of this image. As a matter of fact, a shift of the lens and screen enables us to obtain a direct image of the network, with a possible filtering in the focal plane, i.e. in reciprocal space. The diffraction patterns of networks $R(n, q)$ at rest, with a majority of occupied sites, are quite similar to those of complete networks since, basically, the same main local structures arise in both networks. This similarity is also observed in the diffraction patterns of some rather dense deterministic fractals $[12,13]$. Thus this diffraction pattern is expected to consist of a few points, because of the square lattice of the matrix and of a weak, extended central isotropic spot, because of disordered lacunae. More precisely, the diffraction pattern depends upon the nature of a site (full or not, square or circle) and of its size [7]. Here, as shown in figures 1 and 2 , an occupied site is represented by a full small circle with a diameter $c$ which is one tenth of the distance a between neighbouring sites. This ensures that an 


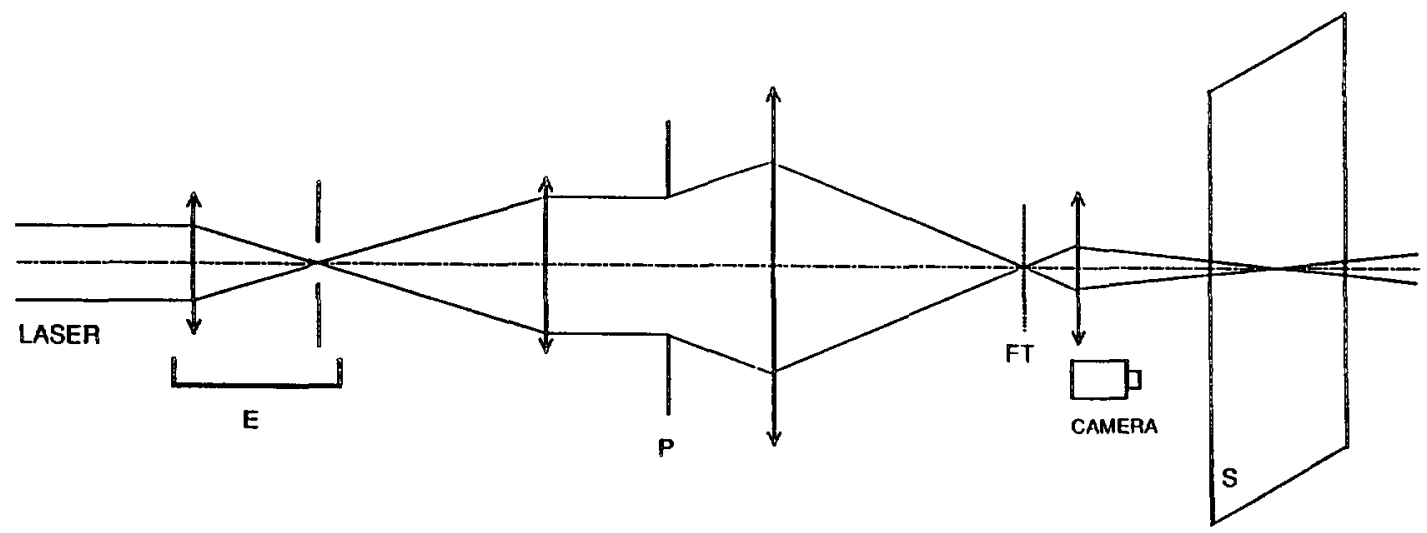

Fig. 4. - Schema of the experiment of optical diffraction.

Airy diffraction pattern of the site is obtained with a central spot which is several times larger than the parameter $b$ of the square lattice in the reciprocal space [7] as observed in figure 5. The diffraction patterns of unachieved random Sierpinski carpets $C(n, p ; k)$ at rest have been investigated previously [7]: the structure of the central isotropic spot enables us to produce evidence for fractal dimension and subdimensions [6] in this spot structure.

Considering the diffraction by a network of points $i$ with a coherent incident radiation of wavevector $\mathbf{k}(0,0, k)$ and a diffracted radiation of wavevector $\mathbf{k}^{\prime}(k x / f, k y / f, k)$, where Gauss conditions imply $x, y \ll f$, the diffraction wavevector $\mathbf{S}$ is : $\mathbf{S}=\mathbf{k}^{\prime}-\mathbf{k}$ and the diffracted intensity $I$ reads :

$$
I=\sum_{i, 1} \exp \left(i \mathbf{S} \cdot \mathbf{r}_{11}\right)=\sum_{1,} \exp \left(i \mathbf{S} \cdot\left[\mathbf{r}_{1}-\mathbf{r}_{1}\right]\right)
$$

the sum is extended over the sites $i$ with the vector $\mathbf{r}_{1}=\mathbf{O M}$ defining the site $i$ in the target plane $\mathrm{O} x y$. For instance a full rectangle $R$ of $N \times P$ sites in the square network is defined by :

$$
\mathbf{r}_{\imath}=n_{1} a \mathbf{i}+p_{1} a \mathbf{j}, \quad 0<n_{1}<N \quad \text { and } \quad 0<p_{1}<P
$$

where $\mathbf{i}$ and $\mathbf{j}$ are the standard unit vectors of orthonormal axes. From equation (4) the diffraction pattern in the focal plane is a square lattice of parameter $b=\lambda(f / a)$, where all points share the same intensity $(N \times P)^{2}$, when $N, P \gg 1$, in the limit $c \ll a$. According to an affine deformation of the initial square lattice into a rectangular lattice, with rectangular parameters $a^{\prime}=(1+\varepsilon) a$ and $a$, as happens with a stress according to the direction $\mathbf{i}$, a rectangular lattice appears in the diffraction pattern, with rectangular parameters $b$ and $b^{\prime}=\lambda\left(f / a^{\prime}\right)$ respectively. It enables us to measure the affinity parameter $(1+\varepsilon)$ and the distortion $\varepsilon$ in a region of the fourth kind from its diffraction pattern; a vernier effect is useful to measure $\varepsilon=1 / u$ by coincidence if $u$ is integer.

The diffraction by several extended undeformed regions I, II and III, which are shifted from each other by arbitrary distances is schematized by that of full rectangles since the diffraction pattern of a full rectangle is only slightly perturbed by the addition of a few lacunae. We consider the diffraction by both a full rectangle $R$ and another independent shifted full rectangle $R^{\prime}$ of $N^{\prime} \times P^{\prime}$ sites. The rectangle $R^{\prime}$ has $\mathbf{M}_{j}$ for running point with $\mathbf{O M}=\mathbf{r}_{j}$ :

$$
\mathbf{r}_{J}=\left(n_{j} a+d\right) \mathbf{i}+\left(p_{J} a+d^{\prime}\right) \mathbf{j}, \quad L<n_{J}<L+N^{\prime} \quad \text { and } \quad L^{\prime}<p_{\jmath}<L^{\prime}+P^{\prime}
$$




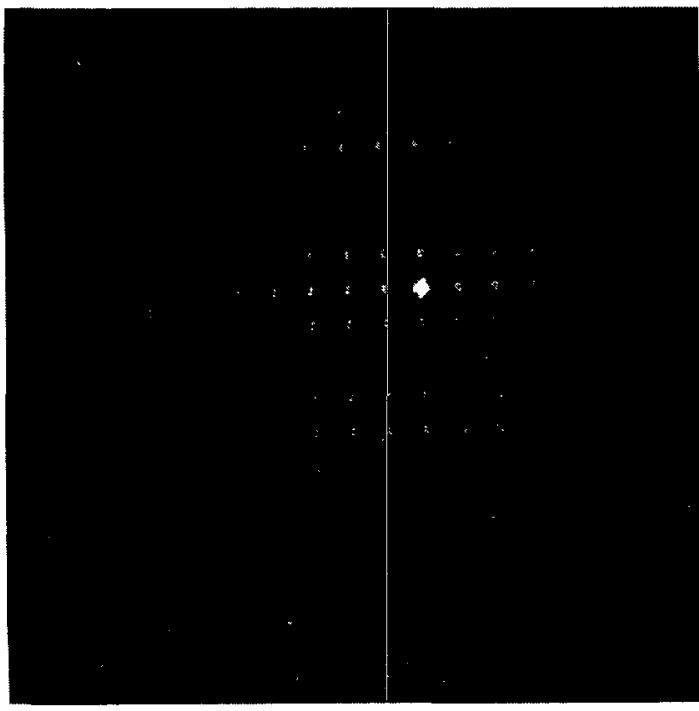

a)

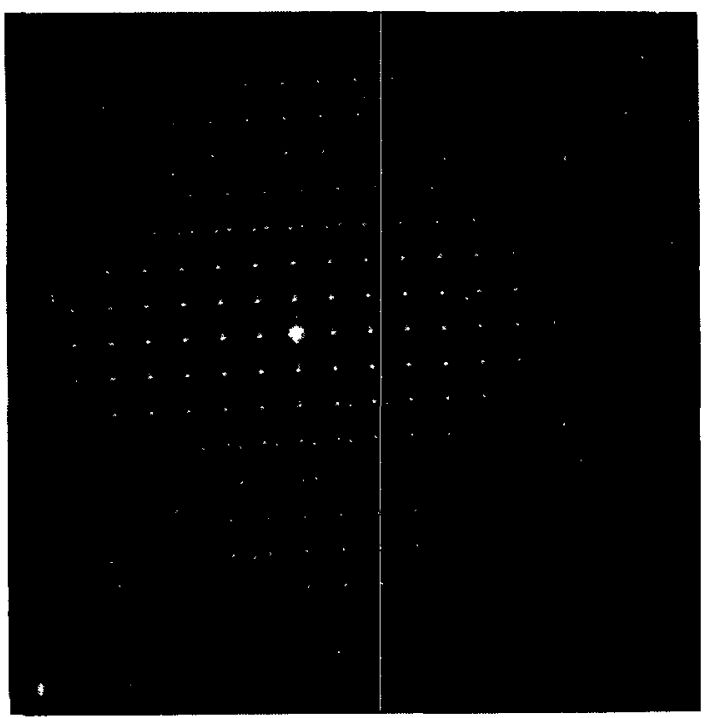

c)

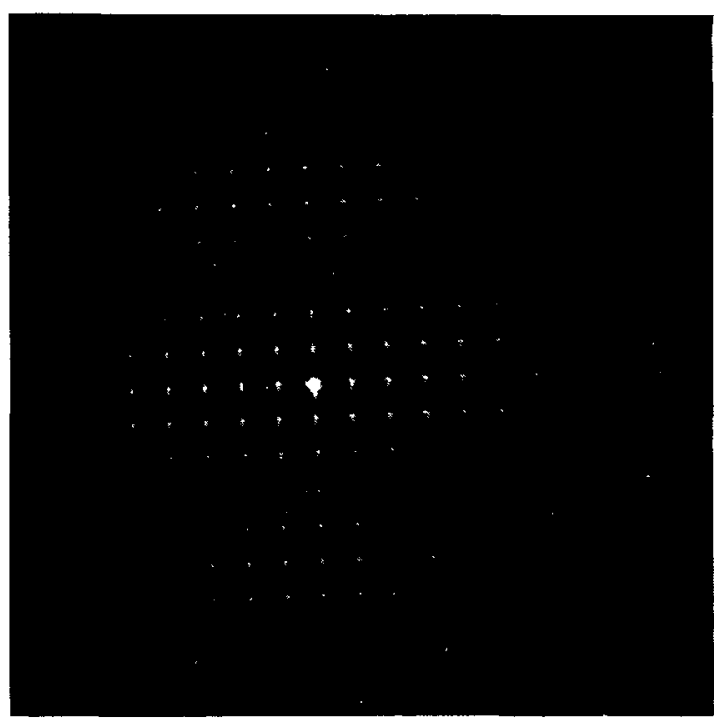

b)

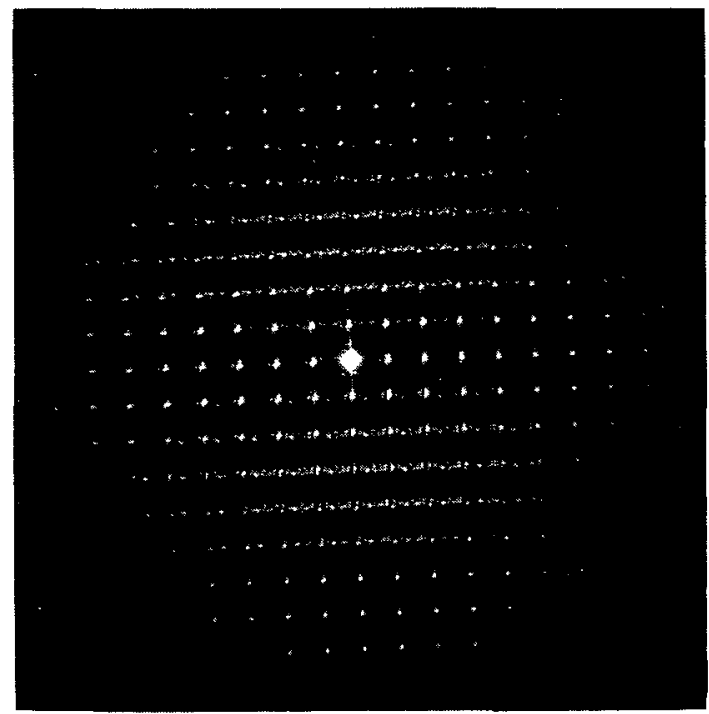

d)

Fig. 5. - Diffraction patterns obtained from pupils such as shown in figure 2 . a) $C(4,13 ; 2)$ with a shift of $0.25 a$ on two external sides, the second row after the central spot is quite black, b) $C(4,13 ; 2)$ with a shift of $0.167 a$ on two external sides, the third row after the central spot is quite black, c) $C(4,13 ; 2)$ with a shift of $0.125 a$ on two external sides, the fourth row after the central spot is quite black, d) the same pupil as in c) but a longer time of exposure : the fourth row after the central spot is a rather bright continuous line as well as adjacent lines.

with a possible non-integer shift in units of $a \mathbf{i}$ and $a \mathbf{j}$ between the two rectangles. The application of equation (4) to both rectangles gives the total diffraction intensity $I$ :

$$
I=(N P)^{2}+\left(N^{\prime} P^{\prime}\right)^{2}+2 N P N^{\prime} P^{\prime} \cos (2 m \pi d / a) \cos \left(2 m^{\prime} \pi d^{\prime} / a\right)
$$


where the attention is restricted to the most illuminated points of the reciprocal space, i.e. those on the square lattice of parameter $b$ with $\left(m, m^{\prime}\right)$ for coordinates where $m$ and $m^{\prime}$ are integers. In the problem of a longitudinal stretch according to the direction $i$, the shift occurs only along the stretch axis, i.e. $d^{\prime}=0$, thus equation (5) means a monodimensional wavy modulation of the intensities on the square lattice of parameter $b$ as observed in figure 5. The wavelength of the modulation gives exactly the non-integer part of the shift $d$ which is experimentally chosen to be in a simple ratio with $a, d=a / 6$ or $a / 4$, in order to obtain a modulation wavelength easily commensurable with $b$. Moreover, in the stress of a random material, the distributed sizes of disconnected blocks, i.e. disconnected segments or groups of disconnected segments, are expected to be quite similar, i.e. $N P \cong N^{\prime} P^{\prime}$. Thus the interference between both terms is total as observed experimentally in the examples of figure 5. Therefore, this diffraction experiment under stress enables us both to measure the non-integer part of the shifts and to appreciate the distribution in disconnected blocks. It must be noted that the shift $d$ occurs between blocks I and III or blocks II and III, but that the shift between blocks I and II is $2 d$. This last shift is less sensitive in the diffraction pattern of figure 5 where the target is specially rich in blocks II because of its fractal nature. Thus the main modulation to be observed in the diffraction pattern has for wavelength $[d / a-E(d / a)]^{-1}$ in units of $b$, where $E(x)$ is the integer part of $x$.

In order to introduce the numerous transverse displacements which are exhibited in the typical displacement vector field given in figure 3 , we consider the diffraction due to a single line of length $L$ where individual sites have uncorrelated transverse displacements. Of course the diffraction of the whole real pattern is more complex, but basic results can be deduced from this simple model where the running point $M_{1}$ has for coordinates $\left(n_{i} a, \xi, a\right)$ when the single line at rest has an ordinate 0 :

$$
\mathbf{O} \mathbf{M}_{1}=\mathbf{r}_{1}=n_{1} a \mathbf{i}+\xi_{1} a \mathbf{j}
$$

where the $n_{l}$ s are successive integers and the $\xi$,'s are uncorrelated numbers, usually smaller than unity. As a matter of fact, a non-zero correlation length appears for sites of a full segment, as observed in figure 3. But, when considering several independent segments, these internal transverse displacements become statistically less correlated, so that the limit over a large number of independent segments is an effective null correlation of internal transverse displacements. In the intensity calculation, the basic scalar product is :

$$
\mathbf{S} \cdot\left(\mathbf{r}_{j}-\mathbf{r}_{1}\right)=\left(n_{J}-n_{l}\right) k a x / f+\left(\xi_{J}-\xi_{l}\right) k a y / f
$$

with the notation $v=k a x / f$ and $v^{\prime}=k a y / f$, the intensity $I$ reads :

$$
\begin{aligned}
I & =\sum_{l, 1} \exp \left[i\left(n_{J}-n_{l}\right) v\right] \exp \left[i\left(\xi_{J}-\xi_{l}\right) v^{\prime}\right] \\
& =\sum_{J, l} \exp \left[i\left(n_{J}-n_{l}\right) v\right] \times \sum_{J, l} \exp \left[i\left(\xi_{J}-\xi_{l}\right) v^{\prime}\right]
\end{aligned}
$$

where the latter form (6b) is due to the uncorrelation of random transverse displacements and means a vertical line $x=m \lambda(f / a)$ with $m$ integer and $y$ arbitrary. Of course, such lines are observed in details in figure $5 \mathrm{c}$. The same total intensity is diffracted by a segment without any transverse displacement as by a segment with numerous transverse displacements. In the first case this intensity is divided into a few diffraction spots (about ten) and in the second case this intensity is distributed over a continuum (practically hundreds of points). Thus this shape of line disappears in the continuum noise if, at the place $x$ of this line, there are simultaneously a few bright diffraction spots because of the modulation given in equation (5). It appears if at this 
phase $x$ there is no diffraction spot because of a black part of this modulation. This is exactly observed in figures $5 \mathrm{c}$ and $5 \mathrm{~d}$ where different times of exposure have been used ; at short times of exposure the modulation is total with an absence of aligned points, while for long times of exposure at the same place, weakly bright, continuous lines appear.

Finally, a direct, rebuilt image of the target, i.e. a second copy of the stressed incomplete network, can be obtained after different possible filterings in reciprocal space. Here, in these preliminary experiments, the central diffracting spot is avoided and some lines of the diffraction spectrum are selected. Some «filtered » images are reported in figure 6 when selecting different such lines in the diffraction pattern. The previous argument which leads to equations (6), means that such a filtering enhances the contribution of transverse distortions, i.e. localized defects, as observed in figure 6 . A more refined selection of the filtering process enables one to introduce arbitrary phase shifts and weights between diffracted components with, for instance, the possibility of carefully selecting the symmetry of the localized defect. Already the simple analysis used here enables us to select different defects.

\section{Conclusion.}

From this work on optical diffraction over pupils deduced from a numerical simulation of stretch in lacunary materials with a central force Hamiltonian it appears that small shifts of disconnected parts can be measured with a high level of accuracy by means of this technique and more generally by diffraction with suitable wavelengths. Moreover, the densities of such disconnected materials can be reached during this approach. The correlation of transverse motions or displacements is also shown to lead to observable effects and finally a fine

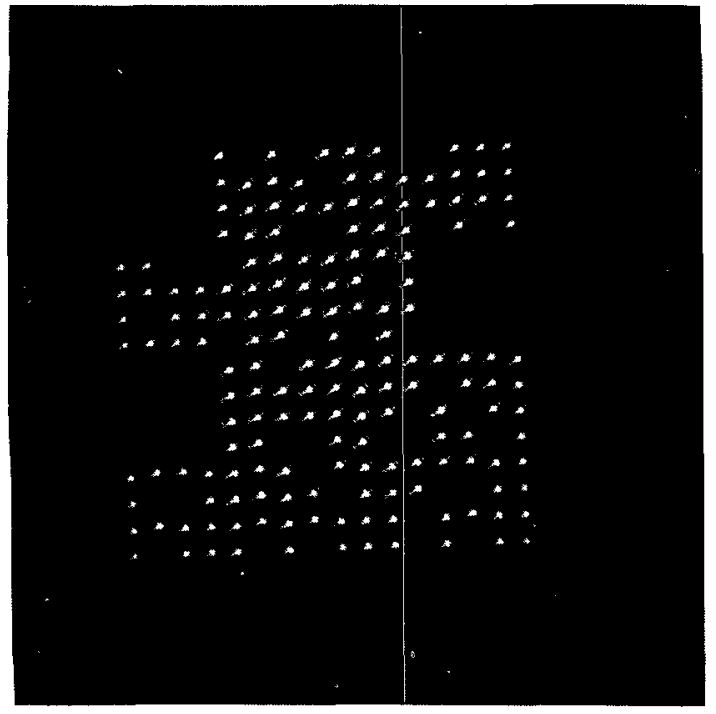

a)

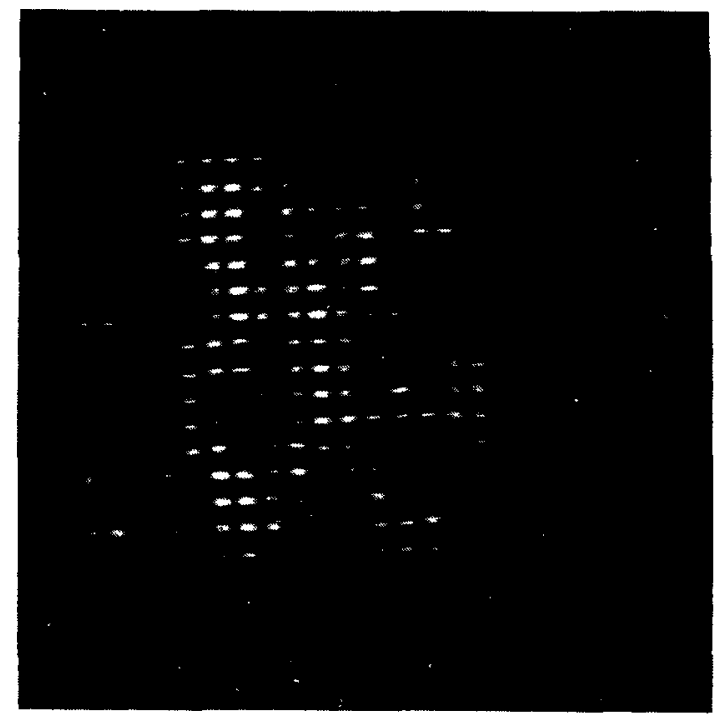

b)

Fig. 6. - Re-built pupils from stretched networks. a) $C(4,13 ; 2$ ) with a shift of 0.125 a on two external sides without filtering, b) the same pupil as in figure 6 a but with only two rows selected in reciprocal space : only rows with transverse motion appear, c) $R(16,6)$ with a shift of $0.3 a$ on two external sides without filtering, d) the same pupil as in figure $6 \mathrm{c}$ but with only two rows selected in reciprocal space : only rows with transverse motion appear. 


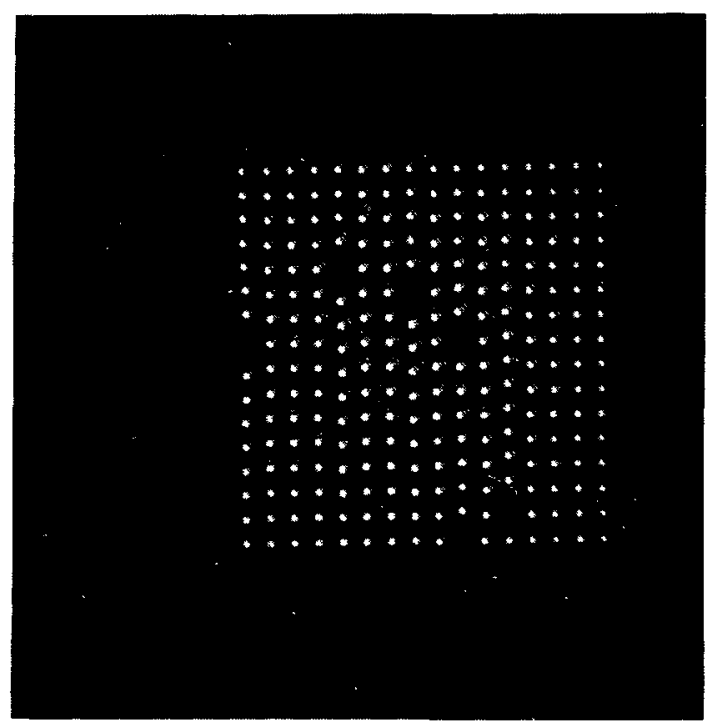

c)

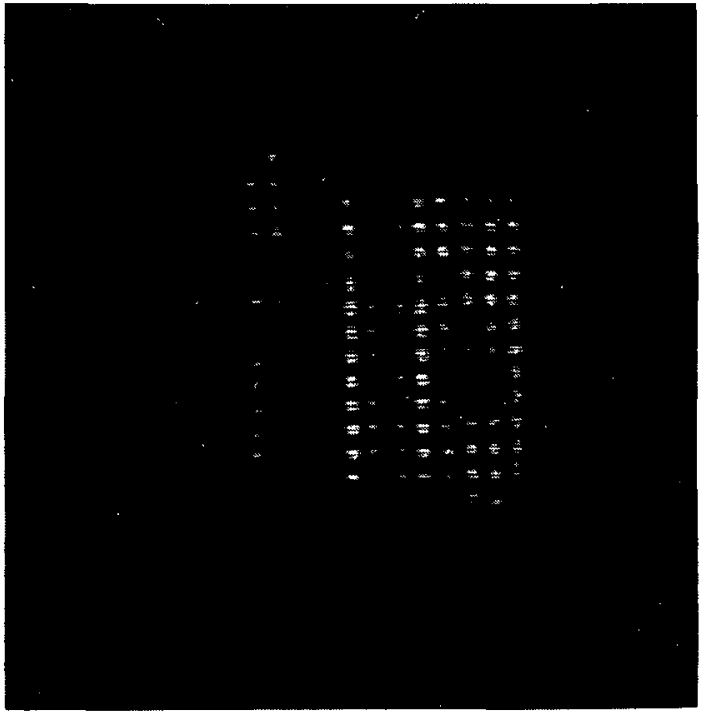

d)

Fig. 6 (continued).

observation of localized defects seems conceivable by means of standard diffraction techniques.

\section{References}

[1] Webman I., Fractals in physics, L. Pietronero and E. Tosatti Eds. (Elsevier Amsterdam, 1986) p. 343.

[2] Oudet C., Kausch H. H., J. Mater. Sci. Lett. (UK) 10 (1991) 1061.

[3] Dorfmüller T., Stellbrink J., Ber. Bunsenges Phys. Chem 95 (1991) 1100.

[4] Monceau P., Lévy J. C. S., submitted for publication.

[5] Wang J., Brooks Harris A., Phys Rev. Lett. 55 (1985) 2459.

[6] Perreau M. and Lévy J. C. S., Phys. Rev. A 40 (1989) 4690.

[7] Berger D., Chamaly S., Perreau M., Mercier D., Monceau P. and Lévy J. C. S., J. Phys. I France 1 (1991) 1433.

[8] Monceau P., Lévy J. C. S. and Perreau M., Phys. Lett. A 171 (1992) 167.

[9] Mercier D. and Lévy J. C. S., Phys. Rev. B 27 (1983) 1292.

[10] Roth J., Schilling R. and Trebin H. R., Phvs Rev. B 41 (1990) 2735.

[11] Hecht E., Optics (Addison Wesley editors, 2nd edition, Reading, Mass., 1987).

[12] Kaye B. H., A random walk through fractal dimension (V.C.H. Verlag, Berlin, 1989).

[13] Allain C., Cloitre M., Physica A 157 (1989) 352. 